\title{
EPENDYMAL STREAKS AND ACCESSORY CAVITIES IN THE HUMAN OCCIPITAL LOBE
}

\author{
BY \\ A. F. LIBER \\ From the Laboratory of Pathology, City Hospital, Department of Hospitals, New York City
}

(ReCEIVED 23RD JULy, 1937)

IT has long been known that the occipital horn is the most variable portion of the lateral ventricles of the human brain. Many authors have alluded to this curious fact. Thus, according to Testut and Latarjet (1929), there are numerous variations between individuals and, in one individual, between the right and the left sides. The different forms of the occipital horn, as observed by Davidoff and Dyke (1935), are as follows : (1) short and stubby ; (2) a straight, fingerlike projection of uniform calibre up to a rounded tip ; (3) tapering almost to a point ; (4) a bulbous tip ; (5) a tip shaped like a fish-tail. Finally, the occipital horn may be completely absent.

In the course of routine examinations of formalin-fixed brains from autopsies at the City Hospital, my attention was attracted to the presence of small, distinct cavities in the occipital lobe, caudal to the tip of the occipital horn of the lateral ventricle.

A search through the available literature revealed only four mentions of such accessory cavities in the occipital lobe. Retzius (1896) merely stated : "Schliesslich kommen auch Fälle vor, wo das hintere Ende des Hinterhorns von der Hauptabteilung abgeschnürt ist und eine besondere, kleine Höhle darstellt." Curran (1909) classified the occipital horns of 64 adult brains into three groups. In his group A the horn was patent to a point beyond the calcar avis ( 22 cases). In group B there was a complete constriction separating the occipital horn from its "isolated open tip" ( 24 cases). In group $\mathrm{C}$ the terminal portion was completely closed, resulting in a short occipital horn (18 cases). The ependyma was stated to persist in the occluded portions. Curran believed that the closure, whether partial or complete, results from the pressure of the rapidly growing calcar in late fotal life. He suggested that ophthalmic migraine, epilepsy, and other transient cerebral disturbances might be due to temporary pressure on a partially closed occipital horn. In the case of a three-week-old infant who died in convulsions, the occipital horns were constricted and the tips were filled with a " cheesy" concretion. In the other cases, nothing was known about the age or history of the subjects. De Angelis (1929) reported the case of a large cavity in the right occipital lobe of a 31-year-old man, who died with a syndrome of intracranial hypertension. The cavity contained clear fluid and compressed the occipital horn, but did not communicate with it. Torkildsen (1934), among 11 cases in which the lateral ventricles were filled with paraffin and subsequently dissected, found one in which the occipital horn "was completely constricted with a cavity posterior to the constriction." No record could be found of microscopic study of these cavities or the surrounding tissues by any of the above-mentioned authors. It would seem difficult to affirm on the basis of gross observation alone that a cavity in the brain is a pinched-off portion of a ventricle and not a cavity secondary to some destructive lesion, such as ischæmic malacia, or to post-mortem change. The latter possibility must be seriously considered in connection with the specimens of Retzius, which were 
fixed in bichromate or in weak formalin, and those of Curran, which came from the dissecting room. Furthermore, Essick (1915) discovered transient juxtaventricular cavities not lined with ependyma in human embryos, and Hochstetter (1919) suggested that in some cases cysts of the adult brain might be persistent embryonal cavities of this sort. This possibility would manifestly have to be considered before affirming that the occipital cavities were portions of the ventricle.

Since the first brain containing accessory occipital cavities was observed at the City Hospital laboratory of pathology, the condition was searched for in all specimens. This was done by making gross coronal or sagittal sections as close together as possible through the formalin-fixed brain. The absence of communication between the separate cavities, when present, and the occipital horn was established in each case by careful inspection, by transillumination, by attempts to pass a filiform bougie, by pouring water into the occipital horn and observing whether or not it passed into the accessory cavity, and finally by attempting to blow air from one cavity to the other while the specimen was submerged in water. It was deemed necessary to carry out these tests in every case, as the occipital horn sometimes narrows down to a minute slit, hardly visible, only to open out again caudally to form a terminal bulb. Without careful examination such forms might be taken for separate cavities. In every case where simple inspection suggested a distinct cavity, this was confirmed by these various tests. In one case with abundant intraventricular hæmorrhage the entire ventricular system was filled with blood, but the accessory cavity contained only clear fluid. In another case, an opaque mass, injected intracisternally, had filled all the ventricles but not the accessory cavity.

Of 50 unselected brains examined with this in view, accessory cavities were present in 16 cases. In five cases the cavities were bilateral. In 11 cases they were unilateral, 10 times on the right side and only once on the left side. The linear measurements of the gross cavities were as follows :

\begin{tabular}{lccc}
\multicolumn{1}{c}{ MEASURE } & AVERAGE & EXTREMES & AVERAGE DEVIATION \\
Rostrocaudal extent. & $5.4 \mathrm{~mm}$. & 8 to $1 \mathrm{~mm}$. & $2.4-$ \\
Transverse extent. & $3.5 \mathrm{~mm}$. & 7 to $1 \mathrm{~mm}$. & 1.5 \\
$\begin{array}{l}\text { Dorsoventral extent. } \\
\text { Distance from tip of }\end{array}$ & $2.9 \mathrm{~mm}$. & 6 to $\frac{1}{2} \mathrm{~mm}$. & $1.4-$ \\
Occipital horn & $10.2 \mathrm{~mm}$. & 15 to $5 \mathrm{~mm}$. & $2 \cdot 1+$
\end{tabular}

The relatively small number of cases precludes a more delicate statistical analysis. The occipital horn was usually short, broad, and relatively blunt-tipped when it was associated with an accessory cavity, longer and narrower in the absence of a cavity. The occipital horns were classified, wherever possible, into long and short types. Of the 50 cases in the series, three (one with and two without gross accessory cavities) had cerebral tumors which deformed the occipital horns beyond the possibility of recognizing their previous shape. In seven further cases, all without cavities, coming from young infants, the occipital horns were of an undifferentiated type which could not be classified as either long or short. This leaves 40 classifiable cases, with 80 occipital horns- 15 cases with cavities and 25 without them. The distribution was as follows :

\footnotetext{
15 CASES WITH GROSS CAVITIES 30 occipital horns :

19 occipital horns with cavities.

11 occipital horns without cavities.

25 CASES WITHOUT GROSS CAVITIES 50 occipital horns.
}

$\begin{array}{cc}\text { SHORT GROUP } & \text { LONG GROUP } \\ 17 & 2 \\ 3 & 8 \\ 21 & 29\end{array}$

The total length of occipital horn, cavity, and intervening tissue was always about equal to that of the contralateral occipital horn in the unilateral cases.

The age-frequency distribution of the 16 cases with accessory occipital cavities 
(6 months to 78 years) and that of the 34 cases without gross cavities (premature stillbirth of 8 months to 84 years) were similar in all but their lowest ranges. Cavities occurred in only one individual less than 16 years of age. This was an infant of 6 months. Of the 34 cases without cavities, one was 14 years old, one was 19 months, one 2 months, three were full-term babies, and two were premature babies, none of whom had accessory cavities. The youngest individual with accessory cavities was 6 months old. The distribution of other factors-sex, race, lesions of the central nervous system, lesions in other organs, cause of death-showed no significant difference between the cases with and those without gross accessory cavities. Of the 50 cases in the series, three presented congenital cerebral anomalies-dilated cavum septi lucidi, ventricular heterotopia of grey matter, congenital tumour of the thalamus, the latter in a new-born baby with arenia, absence of genitalia, and other anomalies. A fourth individual, with congenital atresia of the bile ducts, was a baby of 2 months. None of these four individuals with developmental anomalies had accessory cavities.

The cavities were constantly situated at a short distance lateral and ventral to the calcarine fissure, to which they bore the same relation as the terminal portion of a long and narrow type of occipital horn. In the larger accessory cavities the calcarine fissure corresponded to a fullness in the dorsomedial wall, representing the calcar avis. The collateral eminence was sometimes indicated in the floor of the cavity. The medial wall was thus always more or less convex in the larger cavities. The inferior wall was flat or slightly excavated. The posterior extremity or base was flat or slightly convex and its outline was a curvilinear triangle. Anteriorly, the cavity narrowed to a tapering point or rounded tip. The general shape was that of a triangular pyramid with rounded angles. In one case the rostrocaudal orientation was inverted - that is, the base was rostral and the apex caudal. The smaller cavities were oval.

The lining of the cavities was whitish and smooth and to the naked eye resembled ependyma. Around the cavity there often was an ill-defined gelatinous area of greyish, slightly translucent tissue, softer in consistency than the surrounding white matter. The cavities contained a small amount of clear, colourless fluid.

Microscopically the lining of the accessory cavities was unmistakably ependyma of relatively low-columnar type. In a few cases there were areas where the ependyma had desquamated. The subependymal tissue was made up of a loose, gliofibrillar mesh, interspersed with numerous small groups of aberrant ependymal cells. Some of these groups formed small true rosettes, with a central cavity. The loose subependymal glia, which corresponded to the gross gelatinous layer, merged imperceptibly with the more closely meshed surrounding feltwork of glia and medullated nerve fibres. In the subependymal glia there often were amyloid bodies, similar to those found near the ventricles in many adult and senile brains.

Examination of the tissue between the tip of the occipital horn and the accessory cavity revealed a constant structure whose presence would seem to be of value in explaining the origin of the cavities as well as, to some extent, the variability of the human occipital horn itself. Joining the tip of the ventricle to the cavity was a narrow streak of grey tissue, somewhat softer than the surrounding white matter. In colour and consistency, this streak resembled the tissue about the accessory cavities and subependymal tissue generally. Microscopically, it was a band measuring from $30 \mu$ to $200 \mu$ wide, made up of glial mesh, looser than in surrounding areas and markedly richer in macroglia, while it was poorer in oligodendroglia. Amyloid bodies were often present. Myelin fibres were absent or very rare in the streak, and there was a sharp demarcation between the neighbouring white matter and the non-medullated band. In and near the streak were large veins, all running approximately parallel to it. In the middle of the streak most of the sections showed a single or a double row of cuboidal ependymal cells. Here and there the cell rows gave place to one or more true rosettes of ependymal cells, with a central, round, or oval empty cavity of variable 
size, from $20 \mu$ to $100 \mu$ in diameter. In some places were seen clusters of from two to eight minute rosettes side by side.

The entire structure joining the tip of the ventricle to the accessory cavity, or other homologous structures, will hereafter be denoted the "ependymal streak." A cavity lined with ependyma but distinct from the occipital horn will be called an "accessory occipital cavity," whether it be seen by the naked eye or microscopically.

The majority of adult brains with gross accessory cavities presented a second ependymal streak, dorsal to the first, shorter than it, and not terminated by a gross cavity. In all other respects- the peculiar texture, the rows of ependymal cells, the parallel blood-vessels, the rosettes - the dorsal and the ventral streaks were similar. In some cases, the dorsal streak ended in a microscopical ependymal cavity. The topographical relations of the double ependymal streak can best be understood by considering the form of the tip of the occipital horn. In most of the occipital lobes with gross accessory cavities, the horn was of fishtail type, i.e. it was flattened from side to side and divided into two terminal portions, which may be termed the dorsal and the ventral recess respectively, separated from one another by a fullness in the retroventricular tissue. This disposition is determined by the convergence of four surfaces of different curvatures, different both in sign and degree. The four surfaces are : (1) medially, the calcar avis, always strongly convex ; (2) dorsally, the bulb of the forceps major, smaller than the calcar and always forming a flatter bulge or even a plane surface ; (3) laterally, the tapetum, whose ventricular surface is concave, but far less curved than the calcar ; (4) ventrally, the narrow collateral eminence, either flat or slightly convex. While it is customary to describe the cross-section of the occipital horn as triangular, it is more often quadrilateral at least in its rostral half. The general form of the cavity resulting from this arrangement is that of a vertical concavo-convex lens, with a strongly curved concave medial surface and a slightly curved convex lateral surface. As one follows the occipital horn caudalward on successive coronal sections, one usually sees the floor and the roof flatten out, if they were not already flat at the start. The floor often becomes slightly concave. Its curve is then continuous with that of the lateral wall, but the curvature is greater, so that the combined lateroventral wall is shaped like a capital letter $\mathbf{J}$ with the upright line slightly curved. In any case, the floor and the roof bulge less and less caudalward. At the same time, the medial and lateral walls join at an acute angle. The apex of the calcarine bulge first meets the tapetum, thus subdividing the occipital horn into its dorsal and ventral recesses. Further caudalward, the zone of coalescence of the medial and lateral walls increases in dorsoventral extent, the two recesses narrow and typically end both in a point. The dorsal recess is almost always triangular in cross-section, the base of the triangle being the roof of the ventricle and the apex the meeting-point of the calcar and the tapetum. The ventral recess is triangular when the floor of the ventricle remains convex or flat. In the frequent cases where the caudal portion of the floor is concave, the ventral recess is crescent-shaped, lying between the ventricular floor and the ventral aspect of the calcar. The ventral recess is always longer than the dorsal.

On a coronal section immediately caudal to the tip of the occipital horn, one sees a narrow grey area which has all the gross and microscopical characters of the ependymal streaks and whose form is an exact projection of the caudal extremity of the ventricle with its subependymal tissue. The relations of this structure are the same as those of the tip of the occipital horn. A few millimetres further caudalward, the retroventricular ependymal mass divides into a dorsal and a ventral portion, which continue as the two separate streaks.

Of the 34 brains without gross accessory cavities, seven came from individuals aged 19 months or less and contained no ependymal streaks. Of the remaining 27 , coming from individuals aged 14 to 84 years, all but two presented ependymal streaks caudal to the tip of the occipital horns. In microscopical sections small rosettes were 
often seen in these streaks. Complete serial sections of these areas could not be undertaken because of material limitations. It is possible that rosettes would have been found constantly in the ependymal streaks. In any case, it is evident that the adult brains with and most of those without gross accessory cavities share the same fundamental structure, the ependymal streak, which, like ependymal tissue everywhere and even in the disordered growth of neoplasms, tends to form epithelium-lined cavities. In some cases, the cavities are large enough to be seen with the naked eye ; in other cases they are microscopical.

The tip of the occipital horn, its recesses, and the ependymal streaks present remarkable variations in the adult brains. Aside from the frequent bifid or fish-tail form which I have taken as the type, there are forms in which only one recess, ventral to the calcar, is present. Whether there be one or two recesses, the ventral recess may be very long and narrow. Its cross-section may be rounded, corresponding to the classical digitiform type, or it may be triangular, crescent-shaped, or reduced to a laterally flattened potential cleft. It may broaden terminally into a bulb, which is then the homologue of a gross accessory cavity, the intervening long ventral recess corresponding to a ventral ependymal streak. In some cases the callosal bulb is oriented so as to belong more nearly to the medial wall than to the roof of the ventricle. It is in these cases that the cross-section of the occipital horn approximates a triangle. If, then, the curve of the bulb continues that of the calcar, the dorsal recess is absent or barely indicated, while the occipital horn as a whole tapers to a point corresponding to the ventral recess, which may itself be short or long. Intermediate between this type and the fish-tail is a triangular cavity tapering rapidly to a point ventral to the calcar, but with a slight dorsal depression between the calcar and the bulb. A small dorsal or ventral recess may merge into a long ependymal streak. In the classification of all the adult occipital horns into long and short types, those with long ventral recesses, continuing beyond the rest of the ventricle, were termed long. The bifid type with the ventral recess ending practically at the same level as the rest of the ventricle and the rapidly tapering triangular type were termed short.

In the brains of the five full-term and 8-month babies in the series, in the one 2 months old, and in the one 19 months old the occipital horns were broad, round in cross-section, and ending in a round tip. Of the various eminences characteristic of the region, only the calcar avis was present. Its bulge was far less sharply marked off from the neighbouring portions of the ventricular walls than in the adult. It diminished gradually caudalward until, in a section just rostral to the tip of the ventricle, the calcar was only represented by a slight flattening of the concave medial wall or was entirely absent. Caudal to the tip of the ventricle, no ependymal streaks could be found, either with the naked eye or microscopically. In one baby of 6 months the occipital horn on one side ended in a typical fish-tail with ependymal streaks and a gross accessory cavity.

\section{Discussion}

All of these data suggest a common explanation-the existence in the human embryo of a primary, broad, rounded occipital horn, ending in a round tip. Following the growth of the various structures which indent the occipital horn-calcar avis, callosal bulb, tapetum, collateral eminence, optic radiations-part of the ventricular cavity is obliterated by apposition and concrescence of its walls. This obliteration takes place at an extrauterine period of development, when the ependyma is completely differentiated. Thus, unlike what happens in the early embryonic obliteration of the dorsal portion of the central canal of the spinal cord, the ependyma in the occipital lobe persists as a solid sheet of variable extent between contiguous layers of white matter. In this sheet of ependyma are small cavities, microscopical or visible to the naked eye, which may be either closed-off remnants of the ventricular cavity or else newly formed rosettes in a one-time solid ependymal streak. 
This hypothesis would well explain, as far as it goes, the great variability of the occipital horn in different individuals and the differences between right and left occipital horns in the same individual. Just why the rate of growth of the structure surrounding the occipital horn should be so variable requires an explanation which only further study can supply.

A second hypothesis is that of a cavity following some destructive lesion and secondarily lined with ependyma. There is nothing in favour of this hypothesis. Indeed, cavities accompanying ischæmic malacia, hæmorrhage, compression by tumours, suppuration, etc., never acquire an ependymal lining. Furthermore, it would seem improbable that such cavities should occur so frequently in the same situation. In any case, this hypothesis could not account for the ependymal streaks.

The ependymal streaks and the accessory occipital cavities mark the boundary between important medullated fibre tracts-the bulb and the principal bundle of the forceps major, the tapetum, the stratum calcarinum-and should be able to serve as added landmarks in distinguishing these tracts in the study of secondary degenerations. The ependymal structures are easily recognizable microscopically with different stains, and as a rule, can be seen with the unaided eye.

The number of cases observed (16 in an unselected series of 50 cases) is as yet too small to permit of any statistically valid conclusions with respect to sex and race incidence and the like. It would seem that the presence of gross accessory occipital cavities is not rare. As no constant lesion or malformation is associated with this condition, it must be considered to be one of the many anatomical variants of the occipital horn.

A fact which may be significant is the greater frequency of the short type of occipital horn and of gross accessory cavities on the right side than on the left. According to Engel (quoted from Testut and Latarjet (1929)), the left occipital horn is longer than the right in two-thirds of the cases. In only one case of my series was a unilateral cavity present in the left occipital lobe. It would have been interesting to know whether this individual was left-handed. Unfortunately there was no record of handedness in any of the cases studied. Attempts at seeking anatomical correlates of handedness would be greatly facilitated if all hospitals emulated the simple practice of the Salpêtrière in Paris, where, following the instructions of Professor Georges Guillain, the handedness of each patient is noted on admission.

A final problem suggested by the accessory cavities is the origin of the fluid contained in them. The same problem is presented by the true rosettes in ependymal tumours. No choroid epithelium was observed in any of the accessory occipital cavities. One must perforce suppose either that the fluid reaches the cavity by transudation from the surrounding tissue spaces or that the ependyma of the cavities is endowed with secretory functions, such as Wislocki and Putnam (1924) demonstrated in the area postrema of the fourth ventricle. In either case it is difficult to imagine any adequate mechanism of absorption of the contained fluid. The ependyma is not flattened, as it would be if subjected to prolonged pressure. It must therefore be supposed either that the fluid is produced slowly enough to be removed by the slight absorptive capacity of the ependyma itself or else that after the fluid fills the cavity no further production of fluid takes place.

\section{Summary}

Small cavities caudal to the tip of the posterior portion of the lateral ventricle were observed in one or both occipital lobes in 16 out of 50 unselected human brains, more frequently in the right than in the left hemisphere. Microscopically these cavities were lined with ependyma.

Between the cavity and the tip of the occipital horn was a grey sheet or streak, shaped in cross-section like a projection of the end of the ventricle. Microscopically 
this streak was a single or a double row of ependymal cells flanked by tissue of subependymal type, with blood vessels parallel to the ependyma. In places the solid ependymal layer was replaced by ependyma-lined microscopic cavities which were homologous to the gross cavities.

In many occipital lobes without gross cavities an ependymal streak and microscopic cavities were found.

In new-born babies and rarely in adults the occipital horn was rounded and there was no ependymal streak or accessory cavity.

In most adults the occipital horn was flattened by the calcar avis and the bulb of the forceps major, suggesting that in extrauterine life the tip of the occipital horn is occluded to a variable extent by the growth of the surrounding tissues, thus giving rise to the ependymal streak. This hypothesis would also explain the great variability of size and conformation of the adult occipital horn.

My indebtedness is due to Dr. James R. Lisa, Pathologist at the City Hospital, for placing the material and the records at my disposal.

\section{REFERENCES}

Testut, J., and Latarjet, A. (1929). Traité d'anatomie humaine. 8th ed., Vol. 2, fasc. 2. Davidoff, L. M., and Dyke, C. G. (1935). Bull. Neurol. Inst. N.Y., 4, 221.

Retzius, M. G. (1896). Das Menschenhirn. Vol. 1. Stockholm.

Curran, E. J. (1909). Boston Med. Surg. J., 161, 777.

Angelis, E. de (1929). Riv. oto-neuro-ophthalm., 6, 507.

Torkildsen, A. (1934). J. Anat. Med., 68, 480.

Essick, C. R. (1915). Contr. Embryol. Carneg. Instn., 2, No. 6, 97.

Hochstetter, F. (1919). Beiträge zur Entwicklungsgeschichte des menschlichen Gehirns. I Teil.

Wislocki, G. B., and Putnam, T. J. (1924).'Anat. Rec., 27, 151. 

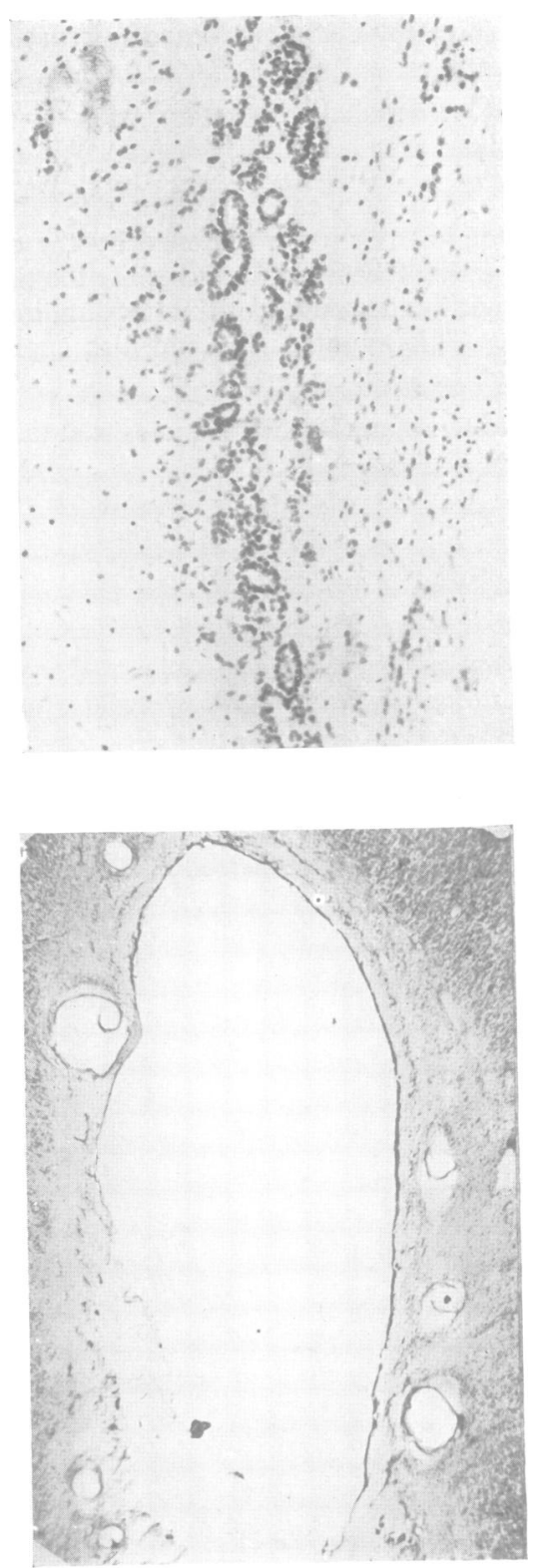

Fig. 1.-Bottom : an ependymal streak at $14 \mathrm{~mm}$. caudal to the ventricular tip, showing solid epithelial rows and multiple rosettes. This case had no gross accessory cavities. Sagittal : $\times 150$ Top: an accessory occipital cavity from a different case. Transverse. The ependyma (dark layer) is desquamated over about one-third of the cavity. The thin-walled blood vessels in the subependymal tissue are all cut transversely, i.e. they are parallel to the direction of the ependymal streak; $\times 20$. 

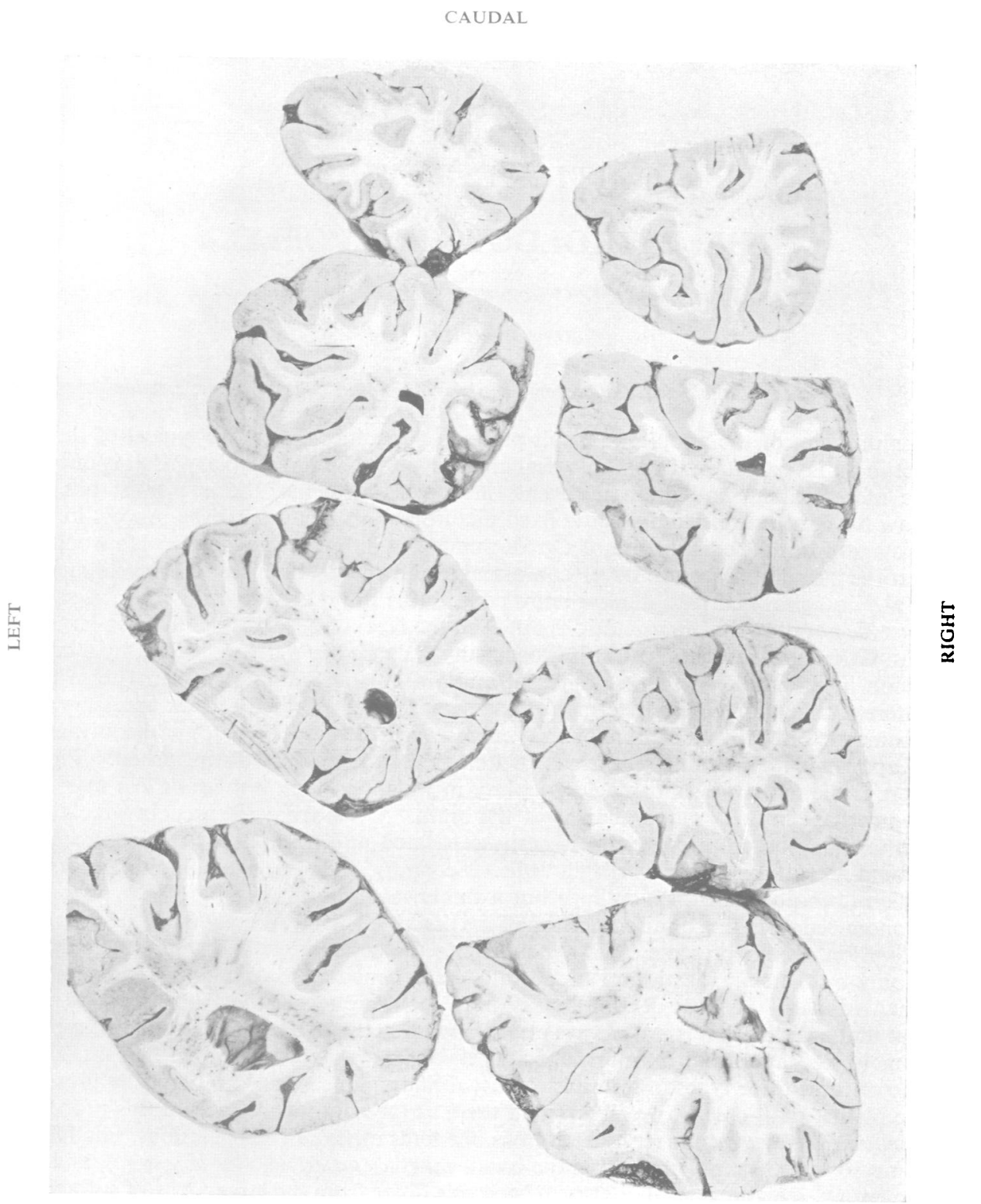

ROSTRAL

Fig. 2.- Rostral aspect of successive coronal slabs of the occipital lobes of an adult human brain. The left occipital lobe has a long and undifferentiated type of posterior horn, ending in a rounded tip. The right horn is of the short fishtail type, indented by the bulb, the calcar, the collateral eminence, and, in this case, secondary eminences. Note the two recesses, of which the ventral is deeper than the dorsal. The second slab shows the grey ependymal streak, between the stratum calcarinum medially and, laterally, the tapetum and optic radiations. The third section shows the caudal segment of the accessory cavity. All ependymal structures are absent from the fourth section. 